\title{
(6) OPEN ACCESS \\ Vitrectomy with and without encircling band for pseudophakic retinal detachment: VIPER Study Report No 2-main results
}

\author{
Peter Walter, ${ }^{1}$ Martin Hellmich, ${ }^{2}$ Sabine Baumgarten, ${ }^{1}$ Petra Schiller, ${ }^{2}$ \\ Endrik Limburg, ${ }^{3}$ Hansjürgen Agostini, ${ }^{4}$ Amelie Pielen, ${ }^{4,5}$ Horst Helbig, ${ }^{6}$ \\ Albrecht Lommatzsch, ${ }^{7}$ Gernot Rössler, ${ }^{1}$ Babac Mazinani, ${ }^{1}$ for the VIPER Study Group
}

- Additional material is published online only. To view please visit the journal online (http://dx.doi.org/10.1136/ bjophthalmol-2016-309240).

'Department of Ophthalmology, RWTH Aachen University,

Aachen, Germany

${ }^{2}$ Institute of Medical Statistics, Informatics and Epidemiology, University of Cologne, Cologne, Germany

${ }^{3}$ Clinical Trials Centre Cologne (CTCC), University of Cologne, Cologne, Germany ${ }^{4}$ Eye Center, Albert-LudwigsUniversity of Freiburg, Freiburg, Germany

${ }^{5}$ Eye Hospital, Hannover Medical School, Hannover, Germany ${ }^{6}$ Department of Ophthalmology, University of Regensburg, Regensburg, Germany ${ }^{7}$ Department of Ophthalmology, Franziskus Hospital, Münster, Germany

\section{Correspondence to}

Dr Babac Mazinani, Department of Ophthalmology, RWTH

Aachen University, Pauwelsstr. 30, Aachen 52074, Germany; bmazinani@gmx.de

Received 24 June 2016 Revised 3 August 2016 Accepted 16 August 2016 Published Online First 8 September 2016

\section{ABSTRACT}

Background It is unclear whether or not an additional encircling band improves outcome in vitrectomy for pseudophakic retinal detachment (PRD). Also unclear is whether small gauge transconjunctival trocar-guided vitrectomy is as successful as conventional 20 gauge (G) vitrectomy.

Methods 257 adult patients with uncomplicated PRD were enrolled in 14 vitreoretinal centres across Germany. Contingent on availability of qualified surgeons, eligible patients were randomly assigned either (i) with ratio 1:1 to $20 \mathrm{G}$ vitrectomy plus encircling band (group E1) or 20 $\mathrm{G}$ vitrectomy without any buckle (group C) or (ii) with ratios 1:1:1 to group $E 1, C$ or $23 / 25 \mathrm{G}$ vitrectomy without any buckle (group E2). Treatment success was defined as no indication for any retina reattaching procedure during the follow-up of 6 months.

Results Success was reached in $79.0 \%(=79 / 100$, group E1) versus $73.5 \%(=72 / 98$, group C) $(p=0.558$, OR $1.32,95 \% \mathrm{Cl} 0.65$ to 2.65 . In group E2 $87.7 \%$ $(=50 / 57)$ of patients reached success compared with $78.7 \%$ (=48/61) in group C, demonstrating noninferiority of $E 2$ to $C$ regarding the prespecified margin of 0.8 (OR scale; $p=0.05,0 R 2.17,95 \% \mathrm{Cl} 0.80$ to 5.89). Best corrected visual acuity significantly increased after surgery independent of technique, that is, on average -0.7 (from 1.0 to 0.3 ) logMAR. Patients suffered from a shift in spherical refraction of $-1.0 \mathrm{D}$ in group E1 compared with $-0.1 \mathrm{D}$ in group C. Similarly, intraoperative complications ( $15.2 \%$ vs $8.8 \%$ of patients) and serious adverse events (30.3\% vs $22.5 \%$ of patients) were more frequent in group E1.

Conclusions Vitrectomy with gas is an efficient and safe treatment for uncomplicated PRD. An additional encircling band does not significantly reduce the risk for any second procedure necessary to reattach the retina in $20 \mathrm{G}$ vitrectomy. Small gauge transconjunctival vitrectomy is not inferior to the conventional $20 \mathrm{G}$ technique.

Trial registration number DKRS 00003158, Results.

\section{INTRODUCTION}

To date high-level evidence is lacking regarding the question whether an encircling band in addition to vitrectomy in the treatment of pseudophakic retinal detachment (PRD) improves outcome. While several retrospective or non-randomised studies reported almost complete success of combined surgery ${ }^{12}$ other studies found no benefit of an additional encircling band compared with vitrectomy alone. ${ }^{3-5}$ An ancillary analysis of the randomised SPR study (Scleral buckling versus primary vitrectomy in rhegmatogenous retinal detachment study) which compared vitrectomy and scleral buckling in the treatment of patients with retinal detachment of medium complexity showed that in pseudophakic patients treated with vitrectomy the use of an additional buckle resulted in a significantly lower redetachment rate of $11.4 \%$ vs $40.9 \%$ in patients who were treated by vitrectomy alone. ${ }^{6}$ In contrast an analysis of survey data on 4179 patients with retinal detachments showed a slightly higher failure rate for combined surgery versus vitrectomy alone, ${ }^{7}$ though selection bias, for example, due to favouring an additional buckle in difficult situations, cannot be ruled out.

The Vitrectomy with and without encircling band in the treatment of pseudophakic retinal detachment (VIPER) Study was set up to answer whether the increased invasivity and operation time, the effect on postoperative refraction and blood circulation of the eye ${ }^{8}$ and potential specific complications such as buckle migration and infection due to an additional encircling band is justified by lower redetachment rates compared with vitrectomy with gas endotamponade alone.

With 23, 25 or even 27 gauge $(G)$ transconjunctival vitrectomy comparable anatomical results were reported in case series, chart reviews ${ }^{9-11}$ and in non-randomised comparative studies. ${ }^{12-14}$ However, since high level evidence is again lacking to date, the VIPER Study was designed to test noninferiority of transconjunctival vitrectomy to $20 \mathrm{G}$ vitrectomy with gas and without encircling band in the treatment of PRD.

\section{METHODS}

\section{Study design}

The VIPER Study was designed as a multicentre, randomised clinical trial. The study was registered in the German Register for Clinical Trials under DRKS 00003158 (http://www.germanctr.de). The design of the study was described in detail in the Viper Study Report No $1 .^{15}$ In brief, 14 clinical sites across Germany participated. All centres were experienced in conducting clinical trials according to good clinical practice. The study protocol was approved by each local ethics committee. The tenets of the Declaration of Helsinki were adhered to. Altogether, 34 vitreoretinal surgeons were eligible for this study according to their surgical logs, either for $20 \mathrm{G}$ procedures alone or for both $20 \mathrm{G}$ and $23 / 25 \mathrm{G}$ procedures depending on the number 
of already performed procedures per surgeon. Patients were randomised to one of the following groups: experimental group 1 (E1): $20 \mathrm{G}$ vitrectomy with gas endotamponade with an additional encircling band. Control group (C): $20 \mathrm{G}$ vitrectomy with gas endotamponade alone. Experimental group 2 (E2): 23 or $25 \mathrm{G}$ vitrectomy with gas endotamponade alone. Patients were randomised either with ratio 1:1 between $\mathrm{E} 1$ and $\mathrm{C}$, or with ratios 1:1:1 between $\mathrm{E} 1, \mathrm{C}$ and $\mathrm{E} 2$ dependent on the certification of the individual surgeon. Our primary working hypotheses were that (i) treatment success over 6 months would be achieved more frequently in group E1 than in group C (superiority hypothesis) and (ii) the proportion of failures over 6 months is not higher for group E2 than for group C (noninferiority hypothesis). The follow-up was over 26 weeks with interim assessments after 6 and 12 weeks.

\section{Primary endpoint}

Success was defined as no indication for any procedure to reattach the retina during the follow-up of 26 weeks. Additional procedures (failure) included any buckling surgery, vitrectomy or tamponade such as reinjection of gas or air or any silicone oil filling. Relevant clinical data (including fundus drawings and photographs) were evaluated by a clinical endpoint committee.

Key secondary endpoints included best corrected visual acuity determined with ETDRS charts, refractive status, adverse events such as the new proliferative vitreoretinopathy (PVR), pucker formation and duration of surgery.

\section{Interventions}

In all three treatment groups the procedure was as standardised as possible for a surgical trial across the participating centres as described in the VIPER Study Report No $1 .{ }^{15}$ In brief, all kinds of encircling bands could be used in group E1. All kinds of gas endotamponades were allowed in all treatment groups. Wide field viewing systems were used. Primary use of silicone, circumferential prophylactic laser coagulation or cryopexy was not allowed in any of the treatment groups. Laser coagulation or cryopexy was allowed only to treat breaks or high risk degenerations.

\section{Eligibility criteria}

Patients were included if they presented with PRD at least 3 months after cataract surgery, following informed consent. Patients were excluded in cases of a giant retinal tear, PVR grade $\mathrm{B}$ or C, any intraocular surgery other than cataract surgery, uncontrolled glaucoma, active vascular diseases, malignant intraocular tumours, active uveitis, aphakia and degenerative myopia. Systemic conditions preventing patients from attending the follow-up visits or making it impossible to perform general anaesthesia were also regarded as exclusion criteria.

\section{Statistics}

Three analysis sets are evaluated: (i) intention-to-treat (ITT) set (all trial subjects enrolled, randomised and with surgery performed; analysis is as assigned), (ii) per-protocol (PP) set (all trial subjects treated and observed according to protocol) and (iii) the as-treated (AT) set (all trial subjects enrolled and randomised; analysis as treated).

Groups E1 and C (regarding superiority) were primarily compared in the ITT set. A sensitivity analysis was done in the PP set (see online supplementary material). Groups E2 and C were primarily compared in both analysis sets (ie, ITT and PP), since to evaluate non-inferiority current regulatory guidance recommends to consider both analyses as equally important. ${ }^{16}$ To guard against selection bias, the comparison of E2 versus C can only include patients operated by vitreoretinal surgeons who qualified for all three surgical procedures. The safety endpoints and adverse events were analysed in the AT set only.

Confirmatory analysis of hypothesis 1: superiority of E1 versus $\mathrm{C}$ regarding the primary endpoint was evaluated by the Cochran-Mantel-Haenszel test stratified by surgeon and the corresponding common OR. ${ }^{17}$ Similarly, the exploratory analysis of hypothesis 2-non-inferiority of E2 to $\mathrm{C}$ using a margin of 0.8 (OR)—was performed. A missing primary endpoint was counted as failure. Since both hypotheses address distinct objectives, no correction for type I error inflation due to multiple testing was applied.

For sample size calculation we assumed event proportions of $15 \%$ (E1) vs $35 \%$ (C), thus 82 patients per group were expected to give the corrected $\chi^{2}$ test at least $80 \%$ power at a two-sided significance level of $5 \%$. Accounting for stratification and $10 \%$ attrition percentage, 100 patients were planned to be assigned to arms E1 and C. After reaching this target, recruitment to the whole trial was stopped, accepting that the comparison E2 versus $\mathrm{C}$ would be explorative only.

\section{RESULTS}

\section{Patient recruitment and baseline data}

In the enrolment phase from June 2011 to August 2013, 257 patients with PRD were included, 100 patients were randomly assigned to E1, 57 to E2 and 100 to C. The recruitment, allocation of treatment and information on analysis sets is summarised in figure 1.

In the ITT set the groups appear well balanced (see table 1) except for the study eye in group E2 (more left eyes, $\mathrm{p}=0.038$ ). The male to female ratio is about $3: 1$ which is well known from other studies. ${ }^{13} 1819$

\section{Primary endpoint}

The median follow-up time of patients was 26 weeks in all groups. A missing primary endpoint defined as follow-up $<23$ weeks with no indication of reattaching procedure was considered a treatment failure (ie, number of cases: E1 vs C: 4/ 100 vs $7 / 98$; E2 vs C: $2 / 57$ vs 5/61; see table 2 ).

Hypothesis 1: superiority of E1 versus C. In the ITT set, 79 of 100 eyes treated with vitrectomy plus encircling band (E1) successfully reached the primary endpoint at 6 months compared with 72 of 98 eyes treated with vitrectomy alone (C) ( $79 \%$ vs $73.5 \%, p=0.558$ from Mantel-Haenszel test stratified by surgeon, pooled OR with 95\% CI 1.32 (0.65 to 2.65), see table 2; (stratified) absolute risk reduction $5.0 \%,-9.2 \%$ to 14.5\%). Disregarding insufficient follow-up (ie, $<23$ weeks) counted as failure, in group E1 17\% (=17/100) and in group C $19.4 \%(=19 / 98)$ of patients needed additional reattaching procedures. In most cases additional vitrectomy was performed (E1: 15/100; C: 18/98). In some cases other procedures like additional buckle were done (E1: 1/100; C: 8/98) or a combination of different procedures (E1: 4/100; C: 11/98). Figure 2A shows the cumulative incidence of detected indications of a reattaching procedure over time (Kaplan-Meier curves; HR 0.85, $95 \%$ CI 0.44 to 1.63 ). Note that about $87.5 \%$ of indications were seen within 10 weeks after initial surgery.

Hypothesis 2: non-inferiority of E2 to C. In the ITT set, 50 of 57 patients treated with $23 / 25$ G vitrectomy with gas (E2) eyes successfully reached the primary endpoint at 6 months compared with 48 of 61 patients treated with $20 \mathrm{G}$ vitrectomy with gas alone (C) $(87.7 \%$ vs $78.7 \%)$. Disregarding insufficient follow-up (ie, <23 weeks) counted as failure, 5 out of 57 patients (E2) or 8 of 61 patients (C) needed an additional 


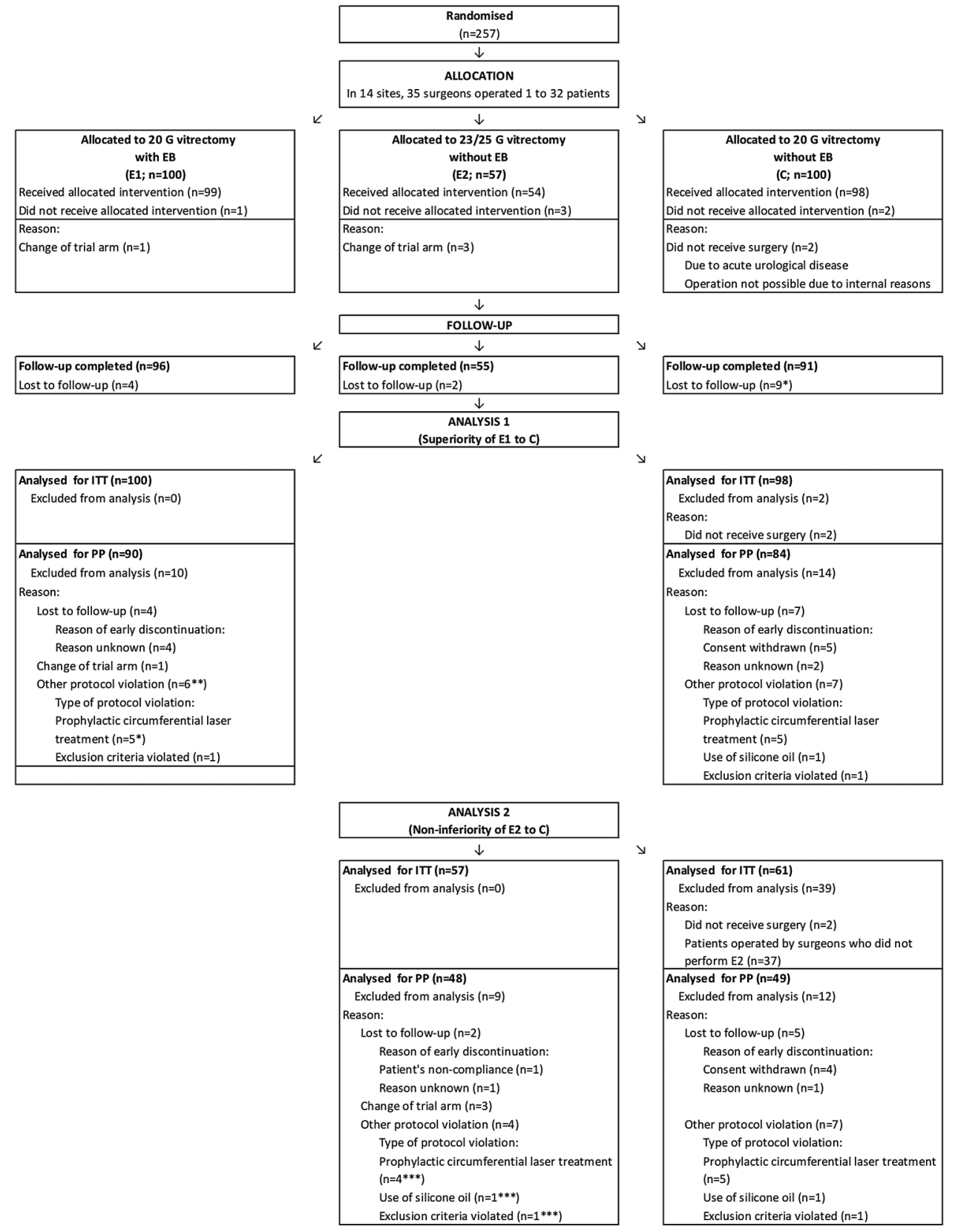

*Inclusive 2 patients that did not receive any surgery

**Patient with change of trial arm had an additional protocol violation

${ }^{* * *}$ One patient with three different protocol violations

Figure 1 Flow of participants. G, gauge; ITT, intention-to-treat; PP, per-protocol.

reattaching procedure $(8.8 \%$ vs $13.1 \%)$. In most cases an additional vitrectomy was performed (E2: 4/57; C: 7/61). In some cases other procedures were done or combined with the additional vitrectomy (see table 2).

The comparison of E2 and C resulted in a pooled OR of 2.17 (95\% CI 0.80 to 5.89). The asymptotic p value for the test of the common OR against the prespecified non-inferiority bound of 0.8 was just $\mathrm{p}=0.05$, that is, non-inferiority of E2 was confirmed. The (stratified) absolute risk reduction of E2 versus $\mathrm{C}$ was $10.2 \%, 95 \% \mathrm{CI}-4.0 \%$ to $16.9 \%$. The cumulative incidence of detected indications of a reattaching procedure over time is shown in figure 2B (Kaplan-Meier curves; HR 0.65, 95\% CI 0.21 to 1.97$)$.

The results of ITT analysis for both comparisons were supported by PP analysis. However, the precision of estimates was lower due to smaller sample size in the PP set (see online supplementary table S3; pooled ORs with 95\% CI: E1 vs C $1.07,0.48$ to 2.38 ; E2 vs C 2.10, 0.51 to 8.61 ).

\section{Secondary endpoints}

Mean visual acuity (in logMAR) equally improved in all groups from baseline to week 26 (between -0.6 and -0.8 , see table 3 and online supplementary table S4). Mean spherical refraction (in D) in group E1 changed significantly by -1.0 (from 0.1 at baseline to -0.9 after 26 weeks), whereas in group $\mathrm{C}$ no significant change was observed during follow-up $(-0.1$ at baseline, -0.2 after 26 weeks; E1 vs C $-0.8(-1.2$ to -0.3$), \mathrm{p}=0.001)$; no relevant difference was observed between groups E2 and C. The proportions of patients with PVR grade C at week 26 were similar in all groups, that is, between $2.1 \%$ and $4.1 \%$. 
Table 1 Description of preoperative characteristics (intention-to-treat set)

\begin{tabular}{|c|c|c|c|}
\hline \multirow[b]{2}{*}{ Characteristic } & \multicolumn{3}{|l|}{ Surgery } \\
\hline & $\begin{array}{l}\text { (E1) } 20 \text { G vitrectomy with } E B(n=100) \\
\text { Summary statistics }\end{array}$ & $\begin{array}{l}\text { (E2) } 23 / 25 \mathrm{G} \text { vitrectomy without } \mathrm{EB}(\mathrm{n}=57) \\
\text { Summary statistics }\end{array}$ & $\begin{array}{l}\text { (C) } 20 \text { G vitrectomy without } E B(n=98) \\
\text { Summary statistics }\end{array}$ \\
\hline Sex, male & $70(70.0 \%)$ & $43(75.4 \%)$ & $71(72.4 \%)$ \\
\hline Age, years & $65 \pm 10$ & $66 \pm 9$ & $64 \pm 10$ \\
\hline Study eye, rightt* & $51(51.0 \%)$ & $20(35.1 \%)$ & $55(56.1 \%)$ \\
\hline Sphere, dioptreł & $0.00(-0.25$ to 0.75$)$ & $0.00(-0.50$ to 0.25$)$ & $0.00(-0.75$ to 0.63$)$ \\
\hline Cylinder, dioptreł & $-0.50(-1.25$ to 0.00$)$ & $-0.25(-0.75$ to 0.00$)$ & $-0.50(-1.13$ to 0.00$)$ \\
\hline Axis, degreeł & 76 (0 to 128$)$ & $45(0$ to 120$)$ & $54(0$ to 112$)$ \\
\hline Intraocular pressure, $\mathrm{mm} \mathrm{Hg}$ & 14 (12 to 16$)$ & 15 (12 to 18$)$ & 15 (12 to 17$)$ \\
\hline Visual acuity, logMAR & $1.1(0.3$ to 1.7$)$ & $1.0(0.5$ to 1.7$)$ & $0.8(0.3$ to 1.7$)$ \\
\hline \multicolumn{4}{|l|}{ Vitreous situation at start of surgery } \\
\hline Fully attached & $6(6.0 \%)$ & $5(8.8 \%)$ & $9(9.2 \%)$ \\
\hline Partly attached & $37(37.0 \%)$ & $22(38.6 \%)$ & $41(41.9 \%)$ \\
\hline Fully detached & $56(56.0 \%)$ & $30(52.6 \%)$ & $44(44.9 \%)$ \\
\hline Haemorrhage & $0(0.0 \%)$ & $0(0.0 \%)$ & $1(1.0 \%)$ \\
\hline Other & $1(1.0 \%)$ & $0(0.0 \%)$ & $3(3.1 \%)$ \\
\hline Cataract surgery uneventful, yesł & $89(89.0 \%)$ & $48(84.2 \%)$ & $89(91.8 \%)$ \\
\hline Laser, yes & $4(4.0 \%)$ & $7(12.3 \%)$ & $8(8.2 \%)$ \\
\hline Cryocoagulation, yes & $1(1.0 \%)$ & $0(0.0 \%)$ & $1(1.0 \%)$ \\
\hline Gas injection, yes & $0(0.0 \%)$ & $0(0.0 \%)$ & $0(0.0 \%)$ \\
\hline Anti-VEGF injection, yes & $0(0.0 \%)$ & $0(0.0 \%)$ & $1(1.0 \%)$ \\
\hline
\end{tabular}

Regarding anatomical success, defined as retina fully attached at week 26, all groups reached percentages of about 95\% (ie, between $93.4 \%$ and $96.0 \%$, see table 3 ).

\section{Subgroup analysis by sex}

As expected, we found no indication of any differential efficacy or safety in women and men. Specifically, regarding the primary endpoint, the OR in men for E1 versus C is 1.26 (95\% CI 0.55 to 2.88) and for E2 versus $C 1.91$ (0.60 to 6.14). In women the OR for E1 versus C is 1.43 (0.30 to 6.86) and for E2 versus C $3.60(0.29$ to 45.11$)$.

\section{Perioperative characteristics}

Median operation time differed by surgery method, $63 \mathrm{~min}$ for $20 \mathrm{G}$ with encircling band (EB) (E1) (25th to 75th percentile $55-80), 38 \mathrm{~min}$ for $23 / 25 \mathrm{G}(\mathrm{E} 2)(33-52)$ and $47 \mathrm{~min}$ for $20 \mathrm{G}$ without EB (C) (37-60; C; E1 vs C: p<0.001; E2 vs C: $\mathrm{p}=0.019$ ), respectively (see online supplementary table $\mathrm{S} 1$ ).

\section{Intraoperative complications}

Thirty-one patients were affected by intraoperative complications (see online supplementary table S1). Most events occurred after $20 \mathrm{G}$ vitrectomy with encircling band (E1, 15.2\%, 15 of 99 patients) followed by $23 / 25 \mathrm{G}$ vitrectomy (E2, 13\%=7/54). The lowest proportion of intraoperative complications were found after standard surgery (20 G vitrectomy without encircling band, $\mathrm{C}, 8.8 \%=9 / 102$ ). Iatrogenic breaks were the most frequently occurring complications during surgery, none in group $\mathrm{C}$ (E1: $6.1 \%=6 / 99$, E2: $9.3 \%=5 / 54, \mathrm{C}: 0 \%=0 / 102 ; \mathrm{p}=0.014)$. The most frequent complication during standard surgery was intraocular vitreous haemorrhage (C: $2.9 \%=3 / 102$, none in E1 and E2).

Table 2 Evaluation of primary outcome, that is, absence of indication for reattaching procedure, based on ITT set (ITT; results for PP set, see online supplementary material)

\begin{tabular}{|c|c|c|c|c|c|c|}
\hline & $\begin{array}{l}\text { E1 } 20 \text { G vitrectomy } \\
\text { with } E B(n=100)\end{array}$ & $\begin{array}{l}\text { C } 20 \text { G vitrectomy } \\
\text { without } E B \quad(n=98)\end{array}$ & $\begin{array}{l}\text { E1 vs C OR } \\
(95 \% \mathrm{Cl})\end{array}$ & $\begin{array}{l}\text { E2 } 23 / 25 \text { G vitrectomy } \\
\text { without EB }(n=57)\end{array}$ & $\begin{array}{l}\text { C } 20 \text { G vitrectomy } \\
\text { without } E B(n=61)\end{array}$ & $\begin{array}{l}\text { E2 vs C OR } \\
(95 \% \mathrm{Cl})\end{array}$ \\
\hline $\begin{array}{l}\text { Absence of indication for } \\
\text { reattaching procedure }\end{array}$ & $79(79.0 \%)$ & $72(73.5 \%)$ & $\begin{array}{l}1.32 \dagger \\
(0.65 \text { to } 2.65)\end{array}$ & $50(87.7 \%)$ & $48(78.7 \%)$ & $\begin{array}{l}2.17 \dagger \star^{*} \\
(0.80 \text { to } 5.89)\end{array}$ \\
\hline Additional vitrectomy & $15(15.0 \%)$ & $18(18.4 \%)$ & - & $4(7.0 \%)$ & $7(11.5 \%)$ & - \\
\hline Additional buckle & $1(1.0 \%)$ & $8(8.2 \%)$ & - & $3(5.3 \%)$ & $4(6.6 \%)$ & - \\
\hline Other procedure & $5(5.0 \%)$ & $4(4.1 \%)$ & - & $1(1.8 \%)$ & $2(3.3 \%)$ & - \\
\hline Follow-up $<23$ weeks§ & $4(4.0 \%)$ & $7(7.1 \%)$ & - & $2(3.5 \%)$ & $5(8.2 \%)$ & - \\
\hline
\end{tabular}

${ }^{*} \mathrm{p} \leq 0.05,{ }^{* *} \mathrm{p} \leq 0.01,{ }^{* * *} \mathrm{p} \leq 0.001$

†Breslow-Day test of homogeneity of ORs over surgeons, $p=0.386(0.071)$.

$¥$ Asymptotic $p$ value for the test of the common OR against 0.8 (non-inferiority bound): $p=0.05$

$\S$ And no indication for reattaching procedure during follow-up.

$E B$, encircling band; $G$, gauge; ITT, intention-to-treat; PP, per-protocol. 

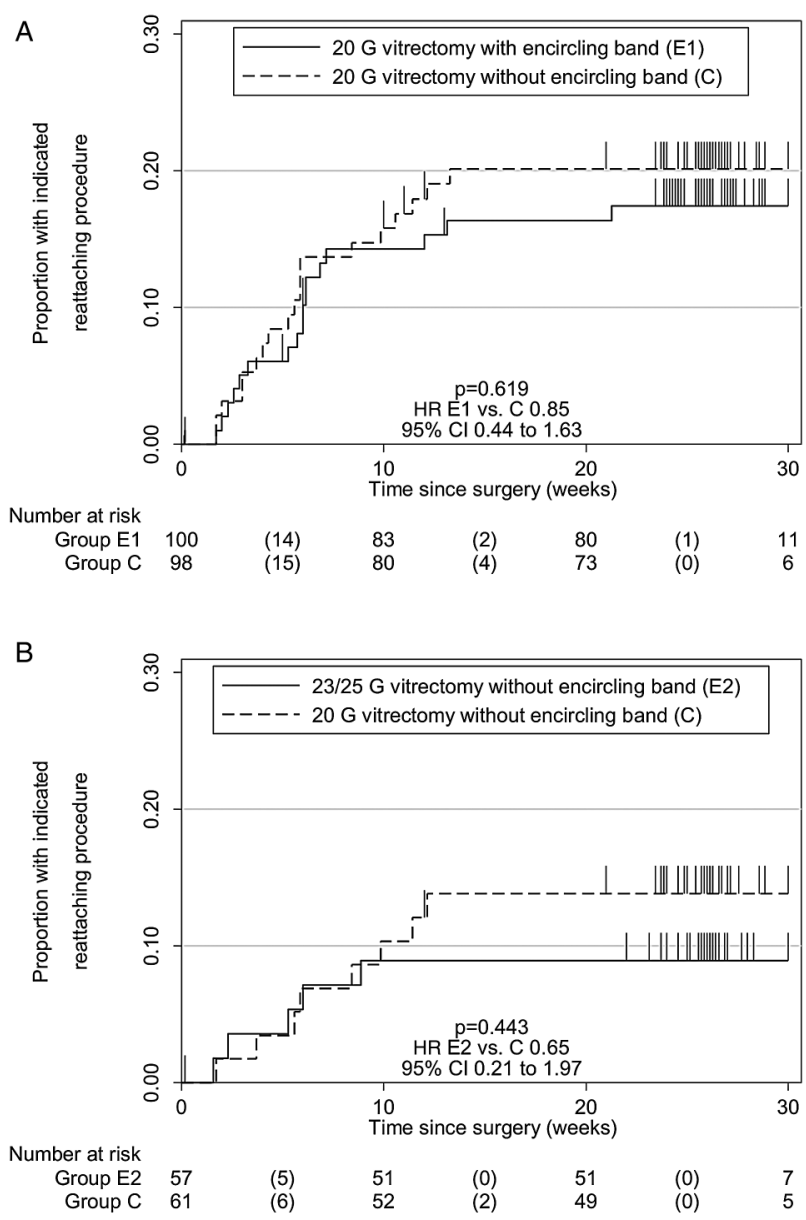

Figure 2 Kaplan-Meier curves for the cumulative proportion without indication for reattaching procedure: $(A)$ treatment $E 1$ versus $C$, (B) treatment E2 versus C (patients with insufficient follow-up, ie, $<23$ weeks, are censored; intention-to-treat set). G, gauge.

After $20 \mathrm{G}$ with encircling band some additional types of intraoperative complications were observed: corneal oedema (4/ $15)$, scleral perforation during buckling and fogged intraocular lens (three in each category). In the other two groups only a few additional individual cases of other types of complications were observed.

\section{Adverse events}

In total, 78 serious adverse events occurred in 60 patients (see online supplementary table S2). In group E1, almost one-third of the patients was affected (E1: $30.3 \%=30 / 99)$, whereas the proportions in group E2 and C were lower (E2: 13\%=7/54; C: $22.5 \%=23 / 102)$. The most frequently observed serious adverse event was retinal redetachment. In total, 52 of those events occurred in 41 patients (E1: 16 of 99 patients, E2: 4/54, C: 21/ 102). Besides, there were some cases of ocular hypertension (3/ 30 patients in E1, none in E2 and C) and pain (2/30, E1) and several individual cases of various types, most of which occurred in group E1.

\section{DISCUSSION}

Retinal surgeons expect additional buckling procedures to provide enhanced support of the vitreous base and better visualisation of the periphery. On the other hand, the additional scleral buckle implies increased invasivity and operation time, it affects the postoperative refraction and the blood circulation of the eye ${ }^{8}$ and may entail specific complications such as buckle migration and infection. In our study the operation duration was significantly longer in the combined surgery of $20 \mathrm{G}$ vitrectomy with additional encircling band (median $63 \mathrm{~min}$ ) than in $20 \mathrm{G}$ vitrectomy alone (47 $\mathrm{min})$ and $23 / 25 \mathrm{G}$ vitrectomy (38 min). This longer operation duration may explain a higher stress on the corneal epithelium which made an abrasion necessary in 4 vs 1 cases in the combined surgery group compared with the $20 \mathrm{G}$ vitrectomy alone group (23/25 G vitrectomy: none). In three cases the sclera was perforated during the fixation of the encircling band. Moreover patients experienced a mean myopisation of $-1.0 \mathrm{D}$ in the combined surgery group versus $-0.1 \mathrm{D}$ in both other groups $(\mathrm{p}<0.001)$.

The literature is inconclusive regarding the question whether these costs of the additional buckle are associated with increased anatomical or functional success (we have provided an overview on existing retrospective and non-randomised prospective studies in the VIPER Study Report No 1). ${ }^{15}$

For the primary comparison of vitrectomy plus encircling band group (E1) versus vitrectomy alone (C) we found a (stratified) absolute risk reduction of 5\% (95\% CI $-9.2 \%$ to $14.5 \%)$ in favour of E1, albeit not statistically significant. In arm E1 $79 \%(79 / 100)$ of patients showed no indication for any retina reattaching procedure compared with $73.5 \%(72 / 98)$ in arm C (Mantel-Haenszel test, stratified by surgeon: $\mathrm{p}=0.558$; pooled OR 1.32 , 95\% CI 0.65 to 2.65 ), see table 2 . Thus, apart from the direction of effect, the large absolute $29.5 \%$ advantage for E1 seen in the SPR trial could not be replicated. The encircling band as an additional means combined with vitrectomy and gas endotamponade does not consistently reduce the risk for any intervention to reattach the retina during a 6-month follow-up. The design of the study implies that the results cannot be merely applied to phakic patients or patients with advanced PVR. However, in phakic retinal detachment primary vitrectomy is only one approach besides buckling ${ }^{6}$ and combined cataract surgery and vitrectomy. ${ }^{20}$ It is unclear if a possible difference in the pathophysiology of PRDs affects conclusions regarding the benefit of additional buckling in these patients.

For the comparison of small gauge transconjunctival vitrectomy (E2) and $20 \mathrm{G}$ vitrectomy with gas alone (C) we demonstrated non-inferiority against the prespecified relative bound of 0.8 (pooled OR 2.17, 95\% CI 0.80 to $5.89, \mathrm{p}=0.05$ ) and, thus, confirmed several retrospective studies who stated 'similar results'. 1214181921 The proportion of patients with no indication of a reattaching procedure was slightly higher in E2 $(87.7 \%$, $50 / 57)$ as compared with $C(78.7 \%, 48 / 61$; see table 2$)$ and the (stratified) absolute risk reduction of E2 versus C was $10.2 \%$, $95 \%$ CI $-4.0 \%$ to $16.9 \%$. Hence, based on our data, absolute differences larger than $-4.0 \%$ in favour of $\mathrm{C}$ can be excluded.

The fact that transconjunctival surgery resulted in less redetachments may be explained by the use of trocar systems which may reduce the risk of applying perpendicular forces to the vitreous base during insertion of instruments, a reduced risk of vitreous incarcerations compared with sclerotomies and a reduced flow through the cutter resulting in a diminished traction on the vitreous base. Accordingly, less retinal detachments have been observed after transconjunctival vitrectomy for macular surgery. 223

As a limitation, the follow-up of 6 months may lead to an overestimation of the success rates because possible redetachments later than 6 months were not identified. However, it is unlikely that a late redetachment after 6 months is still causally related to the initial condition. Data from large case series suggest that redetachment usually occurs within 6 months. In a series by Lee $85 \%$ of redetachments occurred within the first 
Table 3 Evaluation of secondary endpoints visual acuity, sphere and anatomical findings, based on intention-to-treat set (last observation carried forward, mean \pm SD or count (percentage); results for PP set, see online supplementary material)

\begin{tabular}{|c|c|c|c|c|c|c|}
\hline Secondary endpoint & Groupt & Baseline & Week 26 & $\begin{array}{l}\text { Difference } \\
\text { week } 26 \text { - baseline }\end{array}$ & Paired t-test $95 \% \mathrm{Cl}$ & $\begin{array}{l}\text { ANCOVA } \neq, \mathrm{E} 1 / 2 \text { vs } \mathrm{C} \text { mean } \\
\text { difference, } 95 \% \mathrm{Cl}\end{array}$ \\
\hline \multirow[t]{4}{*}{ Visual acuity, logMAR } & E1 $(n=98)$ & $1.0 \pm 0.7$ & $0.3 \pm 0.4$ & $-0.7 \pm 0.7$ & -0.8 to $-0.6^{* * *}$ & \\
\hline & $C(n=97)$ & $0.9 \pm 0.7$ & $0.3 \pm 0.4$ & $-0.6 \pm 0.6$ & -0.8 to $-0.5^{* * *}$ & $0.0(-0.1$ to 0.1$)$ \\
\hline & E2 $(n=55)$ & $1.0 \pm 0.6$ & $0.3 \pm 0.4$ & $-0.8 \pm 0.6$ & -0.9 to $-0.6^{* * *}$ & \\
\hline & $C(n=60)$ & $1.0 \pm 0.7$ & $0.3 \pm 0.3$ & $-0.7 \pm 0.7$ & -0.9 to $-0.6^{* * *}$ & $0.0(-0.1$ to 0.1$)$ \\
\hline \multirow[t]{5}{*}{ Sphere, dioptre } & E1 $(n=97)$ & $0.1 \pm 1.4$ & $-0.9 \pm 1.5$ & $-1.0 \pm 1.9$ & -1.4 to $-0.6^{* * *}$ & \\
\hline & $C(n=96)$ & $-0.1 \pm 1.4$ & $-0.2 \pm 1.9$ & $-0.1 \pm 1.8$ & -0.5 to 0.2 & $-0.8(-1.2 \text { to }-0.3)^{* * *}$ \\
\hline & E2 $(n=55)$ & $-0.1 \pm 1.2$ & $-0.2 \pm 1.3$ & $-0.1 \pm 1.5$ & -0.5 to 0.3 & \\
\hline & $C(n=60)$ & $-0.1 \pm 1.3$ & $-0.2 \pm 1.6$ & $-0.1 \pm 1.4$ & -0.5 to 0.3 & $-0.0(-0.5$ to 0.5$), 0.878$ \\
\hline & Groupt & Baseline & Week 26 & & & $\begin{array}{l}\text { Exact Fisher test, week 26, E1/2 vs } \\
\text { C OR, } 95 \% \mathrm{Cl}\end{array}$ \\
\hline \multirow[t]{4}{*}{ Retina fully attached } & E1 $(n=99)$ & $0(0.0 \%)$ & $95(96.0 \%)$ & & & \\
\hline & $C(n=98)$ & $0(0.0 \%)$ & $94(96.0 \%)$ & & & $1.0(0.2$ to 4.2$)$ \\
\hline & E2 $(n=57)$ & $1(1.8 \%)$ & $54(94.7 \%)$ & & & \\
\hline & $C(n=61)$ & $0(0.0 \%)$ & $57(93.4 \%)$ & & & $1.3(0.3$ to 5.9$)$ \\
\hline \multirow[t]{4}{*}{ Retina PVR grade C } & E1 $(n=97)$ & $0(0.0 \%)$ & $4(4.1 \%)$ & & & \\
\hline & $C(n=96)$ & $1(1.0 \%)$ & $2(2.1 \%)$ & & & $2.0(0.4$ to 11.3$)$ \\
\hline & E2 $(n=53)$ & $1(1.9 \%)$ & $2(3.8 \%)$ & & & \\
\hline & $C(n=59)$ & $1(1.7 \%)$ & $2(3.4 \%)$ & & & $1.1(0.2$ to 8.2$)$ \\
\hline
\end{tabular}

${ }^{*} p \leq 0.05,{ }^{* *} p \leq 0.01,{ }^{* * *} p \leq 0.001$

†Patients with missing baseline value were excluded.

¥Adjusted for baseline value.ANCOVA, analysis of covariance; C, 20 gauge vitrectomy without encircling band (EB); E1, 20 gauge vitrectomy with EB; E2, 23/25 gauge vitrectomy without EB; PP, per-protocol.

3 months and $97.7 \%$ of failure was seen within the first 6 months after the initial procedure. ${ }^{24}$ These results are confirmed by the observations in the VIPER Study: redetachments occurred within 12 weeks after the initial surgery with a small trend towards earlier redetachments when an encircling band was used (see figure 2A).

Consequently, it appears not necessary to place a $360^{\circ}$ buckle in addition to primary vitrectomy with gas endotamponade to successfully treat pseudophakic retinal detachment. The noninferiority analysis showed that small gauge vitrectomy using transconjunctival techniques is at least equally successful and safe and can be recommended as well as the $20 \mathrm{G}$ technique.

Acknowledgements This work was supported by retina.net, the German competence network for clinical studies in retinal disorders. Retina.net provided the communication infrastructure for the VIPER Study Group and the resources for the internet randomisation, statistics as well as the setup and management of the data entry system. More information on retina.net is given under http://www.retina-net. uni-koeln.de.

Collaborators The VIPER Study Group consisted of the following investigators and subinvestigators: PW, BM, GR, HA, B Junker, T NeB, AP, AL, A Joussen, S Aisenbrey, M Partsch, U Bartz-Schmidt, HH, F Holz, B Kirchhof, C Dahlke, S Fauser, A Lappas, N Eter, C Uhlig, U Ritzau-Tondrow, N Feltgen, MA Gamulescu, M Rudolf, M Lüke, N Bornfeld, M Gök, P Wiedemann, C Jochmann, P Meier, A Nestler, W Rasche, C Clemens, J Hillenkamp, H Roider. The authors thank H Heimann for continuous discussion and support for the design of the VIPER Study.

Contributors PW: conception, design, data acquisition, analysis, interpretation, critical revision. MH, PS: conception, design, analysis, drafting. SB: data acquisition, analysis, interpretation, critical revision. EL: conception, data acquisition, analysis, critical revision. $H A, A P, H H, A L, G R$ : data acquisition, analysis, critical revision. BM: design, data acquisition, analysis, interpretation, drafting. All authors: final approval and accountability for all aspects of the work

Funding Bundesministerium für Bildung und Forschung (01KN1106), Dr. Werner Jackstädt-Stiftung, retina.net, Retinologische Gesellschaft, Deutsche Ophthalmologische Gesellschaft.

Competing interests None declared.

Ethics approval Ethics committees of all participating institutions.
Provenance and peer review Not commissioned; externally peer reviewed.

Open Access This is an Open Access article distributed in accordance with the Creative Commons Attribution Non Commercial (CC BY-NC 4.0) license, which permits others to distribute, remix, adapt, build upon this work non-commercially, and license their derivative works on different terms, provided the original work is properly cited and the use is non-commercial. See: http://creativecommons.org/ licenses/by-nc/4.0/

\section{REFERENCES}

1 Desai UR, Strassman IB. Combined pars plana vitrectomy and scleral buckling for pseudophakic and aphakic retinal detachments in which a break is not seen preoperatively. Ophthalmic Surg Lasers 1997;28:718-22.

2 Devenyi RG, de Carvalho Nakamura H. Combined scleral buckle and pars plana vitrectomy as a primary procedure for pseudophakic retinal detachments. Ophthalmic Surg Lasers 1999;30:615-18.

3 Pournaras CJ, Kapetanios AD. Primary vitrectomy for pseudophakic retinal detachment: a prospective non-randomized study. Eur J Ophthalmol 2003; 13:298-306.

4 Stangos AN, Petropoulos IK, Brozou CG, et al. Pars-plana vitrectomy alone vs vitrectomy with scleral buckling for primary rhegmatogenous pseudophakic retinal detachment. Am J Ophthalmol 2004;138:952-8.

5 Wickham L, Connor M, Aylward GW. Vitrectomy and gas for inferior break retinal detachments: are the results comparable to vitrectomy, gas, and scleral buckle? $\mathrm{Br}$ J Ophthalmol 2004;88:1376-9.

6 Heimann H, Bartz-Schmidt KU, Bornfeld N, et al. Scleral buckling versus primary vitrectomy in rhegmatogenous retinal detachment: a prospective randomized multicenter clinical study. Ophthalmology 2007;114:2142-54.

7 Adelman RA, Parnes AJ, Ducournau D, et al. Strategy for the management of uncomplicated retinal detachments: the European vitreo-retinal society retinal detachment study report 1. Ophthalmology 2013;120:1804-8.

8 Ogasawara H, Feke GT, Yoshida A, et al. Retinal blood flow alterations associated with scleral buckling and encircling procedures. Br J Ophthalmol 1992;76:275-9.

9 Figueroa MS, Contreras I, Noval S, et al. Anatomic and visual outcomes of 23-G vitrectomy without scleral buckling for primary rhegmatogenous retinal detachment. Eur J Ophthalmol 2013;23:417-22.

10 Romano MR, Angi M, Valldeperas $X$, et al. Twenty-three-gauge pars plana vitrectomy, Densiron-68, and 360 degrees endolaser versus combined 20-gauge pars plana vitrectomy, scleral buckle, and SF6 for pseudophakic retinal detachment with inferior retinal breaks. Retina (Philadelphia, Pa) 2011;31:686-91. 
11 Tsang CW, Cheung BT, Lam RF, et al. Primary 23-gauge transconjunctival sutureless vitrectomy for rhegmatogenous retinal detachment. Retina (Philadelphia, Pa) 2008;28:1075-81.

12 Albrieux M, Rouberol F, Bernheim D, et al. Comparative study of 23-gauge vitrectomy versus 20 -gauge vitrectomy for the treatment of rhegmatogenous retinal detachment. Graefes Arch Clin Exp Ophthalmol 2011;249:1459-68.

13 Colyer MH, Barazi MK, von Fricken MA. Retrospective comparison of 25-gauge transconjunctival sutureless vitrectomy to 20-gauge vitrectomy for the repair of pseudophakic primary inferior rhegmatogenous retinal detachment. Retina (Philadelphia, Pa) 2010;30:1678-84.

14 Lewis SA, Miller DM, Riemann CD, et al. Comparison of 20-, 23-, and 25-gauge pars plana vitrectomy in pseudophakic rhegmatogenous retinal detachment repair. Ophthalmic Surg Lasers Imaging 2011;42:107-13.

15 Mazinani B, Baumgarten S, Schiller $\mathrm{P}$, et al. Vitrectomy with or without encircling band for pseudophakic retinal detachment: a multi-centre, three-arm, randomised clinical trial. VIPER Study Report No. 1-design and enrolment. Br J Ophthalmol 2016;100:405-10.

16 Committee for Proprietary Medicinal Products. Points to consider on switching between superiority and non-inferiority. Br J Clin Pharmacol 2001;52:223-8.

17 Mantel N, Haenszel W. Statistical aspects of the analysis of data from retrospective studies of disease. J Nat/ Cancer Inst 1959;22:719-48.
18 Dell'Omo R, Barca F, Tan HS, et al. Pars plana vitrectomy for the repair of primary, inferior rhegmatogenous retinal detachment associated to inferior breaks. A comparison of a 25-gauge versus a 20-gauge system. Graefes Arch Clin Exp Ophthalmol 2013;251:485-90.

19 Von Fricken MA, Kunjukunju N, Weber C, et al. 25-Gauge sutureless vitrectomy versus 20-gauge vitrectomy for the repair of primary rhegmatogenous retinal detachment. Retina (Philadelphia, Pa) 2009;29:444-50.

20 Smith M, Raman SV, Pappas G, et al. Phacovitrectomy for primary retinal detachment repair in presbyopes. Retina (Philadelphia, Pa) 2007;27:462-7.

21 Hikichi T, Kosaka S, Takami K, et al. Surgical outcomes of 23- and 20-gauge vitrectomies for rhegmatogenous retinal detachment associated with posterior vitreous detachment. Ophthalmic Surg Lasers Imaging 2011;42:376-82.

22 Le Rouic JF, Becquet F, Ducournau D. Does 23-gauge sutureless vitrectomy modify the risk of postoperative retinal detachment after macular surgery? A comparison with 20-gauge vitrectomy. Retina 2011;31:902-8.

23 Pielen A, Guerra NI, Böhringer D, et al. Intra- and postoperative risks and complications of small-gauge (23-G) versus conventional (20-G) vitrectomy for macular surgery. Eur J Ophthalmol 2014;24:778-85.

24 Lee E, El Housseini Z, Steel DH, et al. An analysis of the outcomes for patients with failed primary vitrectomy for rhegmatogenous retinal detachment. Graefes Arch Clin Exp Ophthalmol 2014;252:1711-16. 